\title{
Manifestaciones orales en pacientes con insuficiencia renal crónica (IRC) sometidos a hemodiálisis: revisión de la literatura
}

\section{Oral manifestations in patients with chronic renal insufficiency (CRI) undergoing hemodialysis: A literature review}

\author{
José Robello Malatto* \\ Jorge Girano Castaños \\ Facultad de Odontología, \\ Universidad de San Martín de Porres, Perú
}

\section{Resumen}

Las enfermedades renales se producen por deterioro y/o alteración de los riñones que impiden su normal funcionamiento; por ello, los pacientes que sufren estas alteraciones son sometidos a diferentes tratamientos de diálisis. Se conoce que enfermedades sistémicas influyen en la presencia de la enfermedad renal crónica y esta a su vez podría generar manifestaciones en la salud general de las personas, incluyendo, por ende, a la cavidad oral. Estudios informan sobre manifestaciones orales que se producen en pacientes diagnosticados con enfermedad renal crónica que se encuentran bajo tratamiento. Las enfermedades orales muchas veces son producto de una mala higiene; sin embargo, las enfermedades sistémicas funcionan como factor de riesgo de enfermedades orales, llegando muchas veces a ser destructivas si es que no se manejan a tiempo y con un correcto diagnóstico. En el presente estudio, se explora la evidencia disponible de manifestaciones orales en pacientes con enfermedad renal crónica bajo tratamiento. El objetivo es controlar y mejorar el diagnóstico diferencial de las manifestaciones orales, así como, apuntar a un mejor tratamiento para la calidad de vida de los pacientes.

Palabras clave: enfermedad renal crónica, insuficiencia renal crónica, manifestaciones orales, enfermedad periodontal.

Este es un artículo Open Access bajo la licencia Creative Commons Atribución-NoComercial-Compartirlgual 4.0 


\begin{abstract}
Kidney diseases are caused by a deterioration and/or alteration of the kidneys that prevent their normal functioning. Therefore, patients who suffer these conditions undergo different dialysis treatments. It is known that systemic diseases affect the body and may cause chronic kidney diseases (CKDs) and these, in turn, could generate manifestations in the general health of people, thus including the oral cavity. Studies report the oral manifestations that occur in patients diagnosed with CKDs who are under treatment. Oral diseases are many times the product of poor hygiene; however, systemic diseases function as a risk factor for oral diseases, which often become destructive if they are not managed on time and with a correct diagnosis. This article aims to search for available evidence of oral manifestations in patients with CKD under treatment. The objective of this research is to control and improve the differential diagnosis of oral manifestations, as well as to find a better treatment for improving the quality of life of the patients.
\end{abstract}

Keywords: chronic kidney disease, chronic renal insufficiency, oral manifestations, periodontal diseases.

\title{
Introducción
}

La insuficiencia renal es una alteración parcial o completa de la función normal del riñón. La enfermedad renal crónica (ERC) se define como una pérdida específica e irreversible de nefronas funcionales, caracterizada por la progresión hacia la enfermedad renal en etapa terminal (ESRD). En general, cuando la función renal disminuye por debajo de aproximadamente el 10\% de la eficiencia normal, la terapia de reemplazo renal es necesaria. ${ }^{1}$

La insuficiencia renal crónica (IRC) es la destrucción pausada, progresiva e inalterable del funcionamiento de los riñones, que son los encargados de eliminar elementos dañinos y desechos metabólicos del organismo. La IRC es la última fase de la enfermedad, que como único tratamiento tiene, necesariamente, a la hemodiálisis, proceso que hace que la salud general empeore y se desencadenen patologías oportunistas en distintas partes del cuerpo. $^{2}$ 
La IRC y la hemodiálisis, como tratamiento, presentan una serie de consecuencias por las diversas alteraciones físicas y metabólicas propias del padecimiento, por lo tanto, la salud bucodental suele ser afectada en diversos aspectos. ${ }^{2}$ En algunos estudios se han reportado la aparición de manifestaciones orales en pacientes con enfermedad renal crónica sometidos a hemodiálisis, dentro de las que se destacan la xerostomía, aliento urémico, alteraciones en la mucosa, placa bacteriana, candidiasis oral y alteraciones dentales. ${ }^{3}$ Actualmente en el Perú, el problema de la IRC no está resuelto debido a que no existen datos referentes a la prevalencia verídica de esta patología, pero se sabe que es un problema de salud pública, por su trascendental morbilidad, mortalidad y costo socioeconómico. ${ }^{4}$

\section{Insuficiencia renal crónica}

La IRC es la pérdida gradual, avanzada e inalterable de las funciones renales, que ocurren cuando los riñones presentan algún tipo de lesión que compromete su correcta función, inicialmente estas lesiones son asintomáticas, y solo se hacen visibles cuando se ha perdido la mitad del funcionamiento normal de los riñones. ${ }^{5}$ También se puede definir como el deterioro estructural o funcional de los riñones, que solo es evidenciado mediante análisis que contienen marcadores de daño durante tres o más meses. ${ }^{6}$

Se clasifica en cinco estadios con el propósito de reconocer a los pacientes con IRC lo más pronto posible, desde el estadio más prematuro, y establecer medidas para retardar su avance, disminuir la morbimortalidad o, en determinados casos, disponer a los pacientes con IRC para la diálisis. ${ }^{6}$

\section{Etiología}

Inicialmente, la inflamación de los glomérulos del riñón, glomerulonefritis, se conocía como el desencadenante principal de la insuficiencia crónica de los riñones, pero en la actualidad, sobre todo en países desarrollados, se conoce que el exceso de glucosa en sangre, presente en pacientes diabéticos, causa daños irreparables en las nefronas. Otra causante de IRC es la nefroesclerosis presente en paciente con hipertensión arterial. ${ }^{6}$ 
La IRC se caracteriza por anomalías a nivel estructural y funcional, que puede presentar una disminución en la tasa de filtración glomerular por pérdida y disminución de la función de las nefronas, que desencadenan una hipertrofia donde las nefronas sobrevivientes intentan compensar la disminución de las nefronas perdidas, a este proceso se le denomina hiperfiltración adaptativa. Cuando este proceso no se da abasto se recurre a la terapia de reemplazo, denominada diálisis, que es vital para mantener la vida del paciente.

En los países con mayor desarrollo existen entre 500 y 1400 pacientes con IRC por cada millón de habitantes; estas cifras aumentan aproximadamente en un 10\% cada año. En Latinoamérica la prevalencia es de 447 personas que padecen de IRC por cada millón de individuos y presentan una incidencia de 147 enfermos por cada millón de individuos. ${ }^{8}$ La sociedad latinoamericana de la especialidad de nefrología, en el año 2013, señaló que existen 650 pacientes diagnosticados con IRC por cada millón de individuos relativamente sanos, propensos a seguir aumentando cada año. Por otra parte, el Instituto Nacional de Estadísticas y Censos de Ecuador, en el año 2014, concluyó que existen aproximadamente 6611 pacientes diagnosticados con IRC. En la actualidad, se considera que existen más de 10000 individuos con IRC, y en la mayoría de los casos se requiere de terapias de sustitución como son las diálisis. ${ }^{9}$ Los pacientes con IRC suelen empeorar debido a que poseen anticuerpos que incrementan el riesgo de presentar efectos secundarios después de la transfusión del flujo sanguíneo. ${ }^{10}$ En el Perú, en un estudio realizado por Gamez, se concluye que existe una prevalencia de $16.8 \%$ de enfermedades renales crónicas en la población; Lima es el lugar con más cantidad de pobladores enfermos, la mayoría mujeres y pacientes con enfermedades sistémicas como diabetes e hipertensión. ${ }^{8}$

\section{Tratamiento}

El tratamiento de hemodiálisis es realizada a través de un instrumento que se encarga de depurar o dializar la sangre desde la arteria del paciente, donde un líquido se mezcla con la sangre para luego retornar al paciente por medio de la cánula de una vena. Esta técnica, en comparación con la diálisis peritoneal, filtra la sangre a una máquina que hace la función de los riñones, 
donde la sangre nociva es eliminada. Sin embargo, este método no reemplaza las principales funciones de los riñones (endocrina y metabólica). ${ }^{11}$ La diálisis peritoneal es un método que se realiza a través del peritoneo y usa una solución denominada dializado. Esta solución aspira los residuos y líquidos del flujo sanguíneo, usando el peritoneo como un filtro, para ello se introduce el dializado en el área peritoneal a través de un catéter y se retira una vez que se ha producido el intercambio de líquidos en el filtro. Se recomienda realizar el procedimiento entre tres y cinco veces al día, según la necesidad del paciente. ${ }^{11}$ Paez et al., clasifican a la IRC como un deterioro progresivo de las funciones del riñón y cuando el riñón funciona al 10\%, como en el caso de insuficiencia renal terminal, es necesario un tratamiento que sustituya al riñón para la supervivencia del paciente. Esto puede ser un trasplante o diálisis peritoneal o hemodiálisis. ${ }^{11}$

Los pacientes sometidos a hemodiálisis refieren que recibir dicho tratamiento implica grandes restricciones a lo largo de sus vida, a diferencia de las personas que reciben diálisis peritoneal, que manejan mejor sus horarios de terapia y tienen menos restricciones, y mayor libertad para cumplir con su vida diaria. ${ }^{12}$

A pesar de los grandes beneficios de este tipo de tratamiento renal, el paciente padece de una serie de alteraciones en el organismo, como son la falta de sueño y apetito, una percepción diferente de los sabores, palidez, falta de energía que lo debilita, ${ }^{13}$ además, alteraciones musculares, que son las que más afectan al paciente ya que disminuye su capacidad física y lo hacen dependiente de otra persona. ${ }^{14}$ Diversos estudios han reportado manifestaciones a nivel de la cavidad oral en pacientes con enfermedad renal crónica sometidos a hemodiálisis, lo que empeora la calidad de vida relacionada a salud oral de los pacientes y su mejora en el tratamiento de las diversas enfermedades sistémicas que podrían presentar. Este suceso suele presentarse con mayor frecuencia en pacientes que presentan, además de la IRC otras enfermedades de tipo sistémicas que empeorarían las condiciones de salud oral. 


\section{Manifestaciones orales}

Los riñones cumplen una de las funciones más importantes del cuerpo humano, la homeostasis, que es el equilibrio químico de los líquidos y electrolitos. Los riñones también tienen una función fundamental a nivel hormonal, ya que participan en la síntesis y metabolismo de la vitamina D, la renina, la eritropoyetina y las prostaglandinas. ${ }^{15,16}$ En estudios realizados en paciente con problemas renales se concluye que hasta el $90 \%$ de la población presenta sintomatología de uremia a nivel oral, como consecuencia de las múltiples alteraciones en los diversos sistemas del organismo provocadas por los daños renales. ${ }^{17,18}$

\section{Sabor y olor urémico}

La halitosis matinal $\mathrm{u}$ olor amoniacal presente en la cavidad oral es uno de los primeros signos que se presentan en pacientes con este tipo de afección; síntoma acompañado de una percepción de sabor amargo o metálico. Estas alteraciones son producidas por un incremento de urea en el flujo salival y su consecuente metabolismo a amoníaco en la cavidad oral. ${ }^{16}$

\section{Sangrado gingival espontáneo}

Los individuos con IRC generalmente padecen de plaquetopenia, fragilidad capilar y adhesión plaquetaria disminuida, dicha alteración es provocada por la intoxicación urémica y al ser sometidos a hemodiálisis estos síntomas se incrementan por la aplicación de heparina y por el daño que sufren las plaquetas al golpearse contra las paredes internas de los conductos del aparato que realiza el intercambio de líquidos, ${ }^{17,19}$ por lo que disminuye la capacidad de coagulación, generando que la hemorragia gingival sea un obstáculo para la atención odontológica. ${ }^{17}$

Como consecuencia de la alteración a nivel de los factores de coagulación el tejido blando de la cavidad oral, como son las encías y mucosas, pueden tomar un color púrpura. ${ }^{20}$ 


\section{Estomatitis urémica}

La estomatitis urémica es una inflamación de la cavidad oral presente en pacientes con enfermedades sistémicas, generalmente va acompañada por úlceras alrededor de toda la mucosa oral, consideradas quemaduras por químicos o por pérdida de la resistencia de los tejidos. ${ }^{16}$ En los pacientes diagnosticados con insuficiencia renal crónica existe un incremento de los niveles de nitrógeno en sangre, lo que ocasiona lesiones rojizas que pueden ser localizadas o generalizadas, cubiertas por una capa de exudado pseudomembranoso de tonos grisáceos, denominados estomatitis urémica. ${ }^{17}$

\section{Xerostomía}

Esta alteración es provocada por el mal funcionamiento de las glándulas salivales. En los pacientes con trastornos de la función renal, como la IRC, este tipo de alteración es parte de las reacciones adversas de los fármacos que conforman el tratamiento como los antihipertensivos. ${ }^{16}$

\section{Equimosis y petequias}

Las discrasias sanguíneas presentes en pacientes con IRC contribuyen a la aparición de lesiones hemorrágicas como equimosis y petequias en la mucosa oral, esto debido a la disminución de los factores de coagulación. ${ }^{21}$

\section{Palidez de las mucosas}

El paciente diagnosticado con insuficiencia renal crónica generalmente presenta disminución de los niveles de hemoglobina, debido a que los riñones producen pocas cantidades de eritoproyetina, falta de producción y en algunos casos lisis de eritrocitos. Todo esto se refleja en el color pálido de las mucosas..$^{19,22}$ 


\section{Glositis}

Las diversas alteraciones a nivel sanguíneo presentes en el trastorno renal, como las trombocitopenias y tromboastenias, generan cambios en la lengua que desencadena en la glositis, considerada inflamación de la lengua acompañada de una sensación de ardor y una mayor sensibilidad a los alimentos. $^{19}$

\section{Hiperplasia gingival}

En pacientes con trastornos renales sometidos a hemodiálisis o con trasplante de uno o ambos riñones, existe un incremento excesivo de la gingiva denominado hiperplasia gingival que es producto de la respiración bucal en pacientes con complicaciones a nivel pulmonar y en pacientes que consumen fármacos, generalmente antihipertensivos. ${ }^{23}$

\section{Antecedentes}

Lecca et al. ${ }^{3}$, en una investigación realizada en el Hospital Alberto Sabogal Sologuren, en el año 2013, plantearon como objetivo determinar las principales manifestaciones presentes en la cavidad oral de pacientes sometidos a hemodiálisis. Se examinó la cavidad oral de 119 pacientes, varones y mujeres, durante los meses de julio y agosto del año 2013, con los siguientes resultados: lengua saburral $8.2 \%$, agrandamiento gingival $63 \%$, sangrado gingival $55.5 \%$, caries dental $85.7 \%$, cálculo dental $80.7 \%$, perdida de inserción dental $72.3 \%$, xerostomía $84 \%$, halitosis $66.4 \%$ y disgeusia $55.5 \%$. Concluyeron que los pacientes sometidos a hemodiálisis en el Hospital Alberto Sabogal Sologuren, presentaron diversas manifestaciones orales, algunas debido a la insuficiencia renal crónica y otras por la hemodiálisis; además, que los tejidos más afectados son los tejidos blandos y las glándulas salivales.

En la investigación de De la Rosa-García et al. ${ }^{9}$, en 2013, se plantearon como objetivo identificar la prevalencia de la candidiasis oral en pacientes diagnosticados con diabetes mellitus, con insuficiencia renal crónica y sin insuficiencia renal crónica. La muestra la conformaron 56 pacientes diabéticos con insuficiencia renal crónica y 118 pacientes diabéticos sin insuficiencia renal crónica. La principal especie de cándida se identificó con API 20C AUX de 
bioMérieux. En los resultados demuestran que la prevalencia de candidiasis oral en paciente diabéticos con insuficiencia renal crónica es de $23.2 \%$ y en pacientes diabéticos sin insuficiencia renal crónica $16 \%, p=0.258$. Concluyen que la mayor diversidad de especie candida no albicans en la cavidad oral se encuentra en paciente diabéticos con insuficiencia renal crónica, debido a que este tipo de pacientes se encuentran más propensos.

Marcatoma Cáceres ${ }^{24}$, en su tesis del año 2017, señala como objetivo determinar las manifestaciones orales presentes en los tejidos duros, blandos y glándulas salivales en niños de 7 a 12 años diagnosticados con insuficiencia renal crónica, atendidos en el área de pediatría del Hospital Edgardo Rebagliati Martins. La población consta de 45 niños, varones y mujeres, entre las edades de 7 a 12 años. Determina que a nivel de los tejidos duros se halla caries dental en el $46.7 \%$ de niños, hipoplasia dental en $44.4 \%$, erosión dental en $53.3 \%$, en cuanto a los tejidos blandos encontró que hay un $66.7 \%$ de niños con gingivitis; también observó que el $22.2 \%$ de niños mantiene una buena higiene oral y un $77.8 \%$ una higiene oral regular. Además, a nivel de las glándulas salivales el $86.7 \%$ de niños tiene un $\mathrm{pH}$ ácido y el $84.4 \%$ presentan halitosis. Concluye que los casos de insuficiencia renal crónica están en aumento y es una preocupación para el área de estomatología por las diversas repercusiones que se presentan en la cavidad bucal.

En 2018, Vizuete M. et al. ${ }^{25}$, establecieron como objetivo identificar la frecuencia de manifestaciones orales a nivel dental y periodontal en pacientes diagnosticados con insuficiencia renal crónica dializados en el centro Nefrology S.A. El estudio se realizó en 62 pacientes con insuficiencia renal crónica bajo tratamiento de hemodiálisis. Se examinó la cavidad oral para observar directamente las manifestaciones orales mediante exámenes periodontales e índices epidemiológicos de morbilidad oral. Concluyeron que la manifestación más frecuente en la cavidad oral son la palidez de la mucosa, periodontitis y un alto índice de placa bacteriana.

Dannewitz et al. ${ }^{26}$, en 2020, evaluaron la prevalencia y la gravedad de la periodontitis en pacientes con enfermedad renal crónica moderada. Analizaron el estado periodontal de 270 pacientes con enfermedad renal crónica moderada seleccionados al azar de una cohorte de 5217 sujetos que participaron en el proyecto prospectivo observacional de enfermedad renal 
crónica alemana (GCKD). Se evaluó el sangrado al sondaje (BOP), la profundidad de bolsillo (PPD) y nivel de inserción clínica (CAL). De acuerdo a los resultados se determina que el $24.4 \%$ de los pacientes con enfermedad renal crónica no muestran signos leves de enfermedad periodontal; el $47.6 \%$ presentan periodontitis moderada y $27 \%$ periodontitis severa. Los datos obtenidos mediante el cuestionario evidencian que el $62.3 \%$ de los sujetos del estudio con periodontitis severa no están al tanto de la presencia de la enfermedad, el $44.4 \%$ niega haber recibido alguna terapia periodontal sistemática hasta el momento, aunque el $50 \%$ de ellos indica que visita a su dentista regularmente para realizar limpiezas dentales profesionales. Los autores concluyen que existe una mayor prevalencia de periodontitis en pacientes con enfermedad renal crónica, y que el conocimiento de la periodontitis es bajo.

También en 2020, Hanim Afzan et al. ${ }^{27}$, evaluaron el estado periodontal de pacientes con enfermedad renal crónica prediálisis. Un total de 46 pacientes con enfermedad renal crónica prediálisis asistieron a la clínica de nefrología en el Hospital Universiti Sains Malasia. El examen periodontal se realizó utilizando la profundidad de sondeo periodontal, pérdida de inserción clínica e índice de placa. Se determina que $74.0 \%$ de los pacientes con enfermedad renal crónica tienen periodontitis leve; $20.0 \%$, periodontitis moderada; y $6 \%$ no tienen periodontitis. Concluyen que existe una mayor prevalencia y gravedad de la periodontitis crónica entre los pacientes con enfermedad renal crónica. Por consiguiente, la salud periodontal de los pacientes con enfermedad renal crónica debe ser examinada y monitoreada.

\section{Conclusiones}

En la actualidad, si bien se encuentran estudios sobre las posibles manifestaciones orales en pacientes con enfermedad renal crónica sometidos a diversos tratamientos, aún es poca la evidencia existente. Es importante para la salud bucal tener un mayor conocimiento sobre estas manifestaciones orales, que se pueden presentar producto de la enfermedad o su tratamiento. Recientemente, en algunos estudios se evidencia la presencia de enfermedades periodontales producto del desorden sistémico de los pacientes, donde se informa que los pacientes con enfermedad renal crónica presentaban 
las peores condiciones orales; las que se incrementan cuando van acompañadas de otras enfermedades sistémicas como la diabetes mellitus. Por lo tanto, se sugiere que la presencia de enfermedades orales en pacientes con IRC ocurre mayormente por otras enfermedades sistémicas que acompañan a la IRC, las que exacerban las manifestaciones y lesiones a nivel de la cavidad bucal. En tal sentido, el control de las diferentes enfermedades sistémicas combinadas con un adecuado control e higiene de la cavidad bucal, puede resultar en una mejor calidad de vida para los pacientes. Es necesario ahondar en investigaciones que aborden ambas enfermedades con la finalidad de contar con mayor evidencia disponible.

\section{Referencias bibliográficas}

1. Miyata Y, Obata Y, Mochizuki Y, Kitamura M, Mitsunari K, Matsuo T, Ohba K, Mukae H, Nishino T, Yoshimura A, Sakai H. Periodontal Disease in Patients Receiving Dialysis. Int J Mol Sci. 2019; 20(15):3805.

2. Rivas-Almonte ÚO, Sacsaquispe-Contreras SJ, Calderón-Ubaqui V. Manifestaciones bucales clínicas y radiológicas en pacientes pediátricos con insuficiencia renal crónica. Artículo de revisión. Odontología Pediátrica. 2014; 13(2):111-8.

3. Lecca M, Meza J, Ríos K. Manifestaciones bucales en pacientes con insuficiencia renal crónica en hemodiálisis. Rev Estomatol Herediana. 2014; 24(3):147-154.

4. Bencomo Rodríguez O. Enfermedad Renal Crónica: prevenirla, mejor que tratarla. Revista Cubana de Medicina General Integral. 2015; 31(3):353-362.

5. Da Silva JW, Rodríguez Ferreira J, Dos Santos Lugão de Souza F, Rodrigues Dos Santos NA, Perígolo Vicente R, Dos Reis Souza R. Infecção de fístula arteriovenosa em pacientes com insuficiência renal crônica: um estudo retrospectivo. Brazilian Journal of Surgery \& Clinical Research. 2018; 24(2):33-39.

6. Torres Zamudio C. Insuficiencia renal crónica. Rev. Med Hered. 2003; 14(1):1-4.

7. Estrada AV, López JAM, Alvarado MR, Cervantes ML. Insuficiencia renal crónica. unidad de proyectos especiales universidad nacional autónoma de méxico. 2009. http://www.medicinaysalud.unam.mx/temas/ 2009/02_feb_2k9.pdf

8. Gámez Jiménez A, Montell Hernández O, Ruano Quintero V, Alfonso de León J, Hay de la Puente Zoto M. Enfermedad renal crónica en el adulto mayor. Rev. Med. Electrón. 2013; 35(4):306-318.

9. De la Rosa-García E., Miramonte M., Bustos J, Mondragón A. Especies de Cándida en candidosis bucal en pacientes diabéticos con y sin insuficiencia renal crónica. Revista ADM. 2013; 70(6):302-308.

10. Herrera-Añazco P, Pacheco-Mendoza J, Taype-Rondan A. La enfermedad renal crónica en el Perú: Una revisión narrativa de los artículos científicos publicados. Acta Medica Peruana. 2016; 33(2):130-7.

11. Páez A, Jofré M, Azpiroz C, De Bortoli A. Ansiedad y depresión en pacientes con insuficiencia renal crónica en tratamiento de diálisis. Rev. Univ. Psychol. 2009; 8(1):117-124.

12. Ruiz de Alegría B, Besabe-Berañano N, Fernandez-Prado E, Baños-Baños C, Nogales-Rodriguez M, Echavarri-Escribano $\mathrm{M}$, et al. Calidad de vida y afrontamiento: diferencias entre los pacientes de diálisis peritoneal continua ambulatoria y hemodiálisis hospitalaria. Enferm. Clin. 2009; 19(2):61-68. 
13. Contreras F, Espinosa J, Esguerra E. Calidad de vida, autoeficacia, estrategias de afrontamiento y adhesión al tratamiento en pacientes con insuficiencia renal crónica sometidos a hemodiálisis. Psicología y Salud. 2008; 18(2):165-179.

14. Oliveros M, Avendaño D, Hursch S, De la Maza M, Pedreros C, Muller H. Estudio piloto sobre entrenamiento físico durante hemodiálisis. Rev. Med. Chile. 2011; 139:1046-1053.

15. López R. Lesiones orales en pacientes trasplantados renales y factores asociados a su aparición [tesis de doctorado]. Universidad Complutense de Madrid; 2008. Disponible en: http://eprints.ucm.es/8187/1/ T30468.pdf

16. Fernández O. Complicaciones odontológicas de la insuficiencia renal crónica. Intramed; 2009. Disponible en: http://www.intramed.net/contenidover.asp?contenidolD=59229

17. Lynch MA, Brightman VJ, Greenberg MS, editores. Medicina bucal de Burket. 9na edición. México: Editorial McGraw-Hill Interamericana; 1996.

18. Plaza Costa A, Silvestre Donat F. Odontología en pacientes especiales. Valencia: Editorial Universidad de Valencia; 2007.

19. Castellanos Suarez JL. Medicina Oral en Odontología. México: Editorial El Manual Moderno; 1996.

20. Díaz, A. Flores F. Hernández J, Pérez C, Jiménez C. Alteraciones bucodentales en niños con insuficiencia renal crónica. 2009; 48(2). Disponible en: http://www.actaodontologica.com/ediciones/2010/2/art9.asp

21. Wood N, Goaz P. Diagnóstico diferencial de las lesiones orales y maxilofaciales. 5ta edición. Madrid: Editorial Hancourt; 1998.

22. Montero S, Basilli A, Castellon. L. Manejo odontológico del paciente con insuficiencia renal crónica. Revista Dental de Chile. 2002; 93(2):13-17.

23. Curto Aguilera A. Manifestaciones orales en la insuficiencia renal. Revista Científica Dental. 2012; 9(1):4953.

24. Marcatoma Cáceres M. Manifestaciones orales en pacientes con insuficiencia renal crónica atendidos en el servicio de pediatría especializada del Hospital Nacional Edgardo Rebagliati Martins, de abril a mayo del 2017 [Tesis para título profesional]. Universidad Alas Peruanas; 2017.

25. Vizuete M, Dona M, Gordon D, Sempertegui D, Sosa D, Singo D. Estado de salud bucal en pacientes con insuficiencia renal crónica bajo tratamiento con hemodiálisis. Revista Odontológica Mexicana. 2018; 22(4):206-213.

26. Dannewitz B, Sommerer C, Stölzel P, Baid-Agrawal S, Nadal J, Bärthlein B, Wanner C, Eckardt E, Zeier M, Schlagenhauf U, Krane V, Jockel-Schneider Y. Estado de la salud periodontal en pacientes alemanes que padecen enfermedad renal crónica: datos del estudio GCKD. J Clin Periodontol. 2020; 47(1):19-29.

27. Afzan Ibrahim H, Karyatee Kassim N, Zahra Jamsari F, Akmar Zainuddin SL, Hafiz Hanafi M, Syazril Adnan A. Salud periodontal de pacientes con enfermedad renal crónica prediálisis en un hospital terciario de Malasia peninsular del noreste. Malasios J Med Sci. 2020; 27(1):106-114. 